\title{
UNSTEADY MHD THERMAL DIFFUSIVE AND RADIATIVE FLUID FLOW PAST A VERTICAL POROUS PLATE WITH CHEMICAL REACTION IN SLIP FLOW REGIME
}

\author{
D. RAVI KUMAR \\ Research Scholar, Department of Mathematics, Rayalaseema Univesity \\ Kurnool-518002, A.P., INDIA \\ E-mail: ravi.jup@gmail.com \\ K. JAYARAMI REDDY \\ Department of Mathematics, K.L. University \\ Vaddeswaram, Guntur-522502, A.P. INDIA \\ M.C. RAJU* \\ Department of Mathematics \\ JNTUA College of Engineering Pulivendula \\ Pulivendula -516 390 A.P. INDIA
}

\begin{abstract}
An analytical solution of an MHD free convective thermal diffusive flow of a viscous, incompressible, electrically conducting and heat-absorbing fluid past a infinite vertical permeable porous plate in the presence of radiation and chemical reaction is presented. The flow is considered under the influence of a magnetic field applied normal to the flow. The plate is assumed to move with a constant velocity in the direction of fluid flow in slip flow regime, while free stream velocity is assumed to follow the exponentially increasing small perturbation law. The velocity, temperature, concentration, skin friction, Nusselt number and Sherwood number distributions are derived and have shown through graphs and tables by using the simple perturbation technique.
\end{abstract}

Key words: MHD, free convection, heat and mass transfer, slip flow regime, thermal diffusion, radiation and chemical reaction.

\section{Introduction}

Free convection arises in the fluids when temperature changes cause density variation leading to buoyancy forces acting on the fluid elements. The most common example of free convection is the atmospheric flow which is driven by temperature differences. Sometimes the flow is also affected by the differences in temperature. This type of flow has applications in many branches of science and engineering. The study of such a flow under the influence of a magnetic field has attracted the interest of many investigators in view of its application in MHD generators, plasma studies, and nuclear reactors. Hydromagnetic heat and mass transfer by natural convection from a permeable surface embedded in a fluid saturated porous medium was studied by Chamkha [1-3]. The fluid under consideration occurs in some chemical reactions, e.g. air and benzene react chemically, so does water and sulphuric acid. During such chemical reactions, there is always generation of heat. Combining heat and mass transfer problems with a chemical reaction have importance in many processes and have therefore received a considerable amount of attention in recent years. In many chemical engineering processes chemical reactions take place between a

* Corresponding author email ; drmcraju@yahoo.co.in 
foreign mass and working fluid which moves due to the stretch of a surface. The order of chemical reactions depends on several factors. One of the simplest chemical reactions is the first- order reaction in which the rate of the reaction is directly proportional to the species concentration. Chemical reactions can be codified as either heterogeneous or homogenous processes. In most cases of chemical reactions the reaction rate depends on the concentration of the species itself. If the rate of reaction is directly proportional to the concentration, then the reaction is said to be a homogeneous reaction. The analysis of the nonlinear MHD flow with heat and mass transfer characteristics of an incompressible, viscous, electrically conducting, and Boussinesq's fluid on a vertical stretching surface with a chemical reaction and thermal stratification effects was made by Kandasamy et al. [4]. The heat and mass transfer characteristics of the natural convection about a vertical surface embedded in a saturated porous medium subjected to a chemical reaction taking into account the Soret and Dufour effect were analyzed by Postelnicu [5]. Hossain [6] studied the effect of radiation on free convection from a porous vertical plate. Ibrahim et al. [7] investigated the effect of the chemical reaction and radiation/absorption on the unsteady MHD free convection flow past a semi-infinite vertical permeable moving plate with the heat source and suction. In many practical applications, the particle adjacent to a solid surface no longer takes the velocity of the surface. The particle at the surface has a finite tangential velocity; it "slips" along the surface. The flow regime is called the slip flow regime and this effect cannot be neglected. Using these assumptions, Sharma and Chaudary [8] discussed the free convection flow past a vertical plate in slip-flow regime and also discussed the free convection flow past a vertical plate in slip-flow regime and also discussed its various applications for engineering purposes. Also, Sharma [9] investigated the effect of periodic heat and mass transfer on the unsteady free convection flow past a vertical flat plate in slip-flow regime when suction velocity oscillates in time. Coupled non-linear partial differential equations governing the free convection flow, heat and mass transfer have been obtained analytically using the perturbation technique. The fluids considered in this investigation are air $(\operatorname{Pr}=0.71)$ and water $(\operatorname{Pr}=7)$ in the presence of hydrogen $(\mathrm{Sc}=0.22)$. Magneto hydro-dynamic convective heat and mass transfer in a boundary layer slip flow past a vertical permeable plate with thermal radiation and chemical reaction were investigated by Pal and Talukdar [10]. Kumar et al. [11] studied the fluctuating flow of an elastic-viscous stratified fluid through a porous medium past an infinite rigid plane in slip flow regime in the presence of a transverse magnetic field. Muthukumaraswamy [12] studied the heat and mass transfer effects on a moving vertical plate in the presence of thermal radiation. Gupta and Sharma [13], Sing et al. [14-16] studied an MHD flow through a porous medium in slip regime. Recently, Jaiswal and Soundalgekar [17] discussed the flow past an infinite vertical plate oscillating in its plane in the presence of a temperature gradient dependent heat source. While Taneja and Jain [18] presented a theoretical analysis for an unsteady free convection flow with radiation in slip flow regime. Yamamoto and Yoshidha [19] studied flow with convective acceleration through a porous medium considering suction and injection. Flow through a plane porous wall and generalization of this study was presented by Yamamoto and Iwamura [20]. Raju et al. [21-23] analyzed the MHD natural convection flow through a porous medium in the presence of thermal diffusion, radiation and Soret effects. Kim et al. [24] studied unsteady MHD convective heat transfer flow past a semi-infinite vertical porous moving plate with variable suction. Daniel et al. [25] discussed thermally driven shallow cavity flows in a porous medium for the intermediate regime and merged layer regime respectively. The object of the present paper is to study the unsteady MHD free convection flow of a viscous fluid past a vertical porous plate embedded in a porous medium in the presence of chemical reaction. In obtaining the solution, the terms containing the radiation effect, temperature gradient dependent heat source are taken into account in the energy equation and chemical reaction. The permeability of the porous medium and the suction velocity are considered to be an exponentially decreasing function of time.

\section{Mathematical formulation}

We consider a two-dimensional unsteady free convection flow of an incompressible viscous fluid past an infinite vertical porous plate. In rectangular Cartesian coordinate system, we take the $x$-axis along the plate in the direction of flow and the $y$-axis normal to it. Further, the flow is considered in the presence of temperature gradient dependent heat source. In the analysis, the magnetic Reynolds number is taken to be 
small so that the induced magnetic field is neglected. Likewise for small velocity, the viscous dissipation and Darcy's dissipation are neglected. The flow in the medium is entirely due to the buoyancy force caused by a temperature difference between the porous plate and the fluid. Under the above assumptions, the equations governing the conservation of mass (continuity), momentum, energy and concentration can be written as follows.

$$
\begin{aligned}
& \frac{\partial v^{\prime}}{\partial y^{\prime}}=0 \\
& \frac{\partial u^{\prime}}{\partial t^{\prime}}+v^{\prime} \frac{\partial u^{\prime}}{\partial y^{\prime}}=g \beta_{T}\left(T^{\prime}-T_{\infty}^{\prime}\right)+g \beta_{C}\left(C^{\prime}-C_{\infty}^{\prime}\right)+v \frac{\partial^{2} u^{\prime}}{\partial y^{\prime 2}}-\frac{v}{k^{\prime}} u^{\prime}-\frac{\sigma B_{0}^{2}}{\rho} u, \\
& \frac{\partial T^{\prime}}{\partial t^{\prime}}+v^{\prime} \frac{\partial T^{\prime}}{\partial y^{\prime}}=\frac{K_{T}}{\rho C_{p}} \frac{\partial^{2} T^{\prime}}{\partial y^{\prime 2}}-\frac{1}{\rho C_{p}} \frac{\partial q_{r}}{\partial y^{\prime}}, \\
& \frac{\partial C^{\prime}}{\partial t^{\prime}}+v^{\prime} \frac{\partial C^{\prime}}{\partial y^{\prime}}=D \frac{\partial^{2} C^{\prime}}{\partial y^{\prime 2}}-K_{C}^{\prime}\left(C^{\prime}-C_{\infty}^{\prime}\right)+D_{1} \frac{\partial^{2} T^{\prime}}{\partial y^{\prime 2}} .
\end{aligned}
$$

The boundary conditions relevant to the problem are

$$
\begin{aligned}
& u^{\prime}=L\left(\frac{\partial u^{\prime}}{\partial y^{\prime}}\right), \quad T^{\prime}=T_{w}^{\prime}, \quad C^{\prime}=C_{w}^{\prime} \quad \text { at } \quad y^{\prime}=0, \\
& u^{\prime} \rightarrow 0, \quad T^{\prime} \rightarrow T_{\infty}^{\prime}, \quad C^{\prime} \rightarrow C_{\infty}^{\prime} \quad \text { as } \quad y^{\prime} \rightarrow \infty
\end{aligned}
$$

where $\quad L_{l}=\frac{2-M_{x}}{M_{x}}$ assume that

The equation of continuity (2.1) yields that $v^{\prime}$ is either a constant or some function of time, hence we

$$
v^{\prime}=-U_{o}\left(1+\varepsilon e^{-n^{\prime} t^{\prime}}\right)
$$

The negative sign indicates that the suction velocity acts towards the plate. Consider the fluid which is optically thin with a relatively low density and the radioactive heat flux is given by Eq.(3.14) as follows

$$
\frac{\partial q_{r}}{\partial y^{\prime}}=4\left(T^{\prime}-T_{\infty}^{\prime}\right) I^{\prime}
$$

The permeability of the porous medium in a non-dimensional form is considered as

$$
k^{\prime}=k_{0}^{\prime}\left(1+\varepsilon e^{-n^{\prime} t^{\prime}}\right)
$$


Now we introduce the following non-dimensional quantities

$$
u=\frac{u^{\prime}}{U_{o}}, \quad y=\frac{y^{\prime} U_{o}}{\mathrm{v}}, \quad n=\frac{4 \mathrm{v} n^{\prime}}{U_{0}^{2}}, \quad t=\frac{U_{o}^{2} t^{\prime}}{4 \mathrm{v}}, \quad T=\frac{T^{\prime}-T_{\infty}^{\prime}}{T_{w}^{\prime}-T_{\infty}^{\prime}}, \quad K=\frac{K_{0}^{\prime} U_{0}^{2}}{\mathrm{v}^{2}}, \quad C=\frac{C^{\prime}-C_{\infty}^{\prime}}{C_{w}^{\prime}-C_{\infty}^{\prime}} .
$$

Introducing Eqs (2.6), (2.7), (2.8) in Eqs (2.2), (2.3), (2.4), we obtain

$$
\begin{aligned}
& \frac{1}{4} \frac{\partial u}{\partial t}-\left(1+\varepsilon e^{-n t}\right) \frac{\partial u}{\partial y}=\operatorname{Gr} T+\operatorname{Gm} C+\frac{\partial^{2} u}{\partial y^{2}}-M_{1} u \\
& \frac{1}{4} \frac{\partial T}{\partial t}-\left(1+\varepsilon e^{-n t}\right) \frac{\partial T}{\partial y}=\frac{1}{\operatorname{Pr}} \frac{\partial^{2} T}{\partial y^{2}}-R T \\
& \frac{1}{4} \frac{\partial C}{\partial t}-\left(1+\varepsilon e^{-n t}\right) \frac{\partial C}{\partial y}=\frac{1}{\operatorname{Sc}} \frac{\partial^{2} C}{\partial y^{2}}-k_{c} C+S_{0} \frac{\partial^{2} T}{\partial y^{2}}
\end{aligned}
$$

and boundary conditions $(2.5)$ reduce to

$$
\begin{aligned}
& u=h\left(\frac{\partial u}{\partial y}\right), \quad T=1, \quad C=1 \quad \text { at } \quad y=0, \\
& u \rightarrow 0, \quad T \rightarrow 0, \quad C \rightarrow 0 \quad \text { at } \quad y \rightarrow \infty \\
& \text { where } \quad \mathrm{Gr}=\frac{g \beta_{T} \cup\left(T_{w}^{\prime}-T_{\infty}^{\prime}\right)}{U_{0}^{3}}, \quad \mathrm{Gm}=\frac{g \beta_{c} \cup\left(C_{w}^{\prime}-C_{\infty}^{\prime}\right)}{U_{0}^{3}}, \quad M_{1}=\frac{1}{k\left(1+\varepsilon e^{-n t}\right)}+M \text {, } \\
& M=\frac{\sigma B_{0}{ }^{2} \mathrm{v}}{v_{0}^{3}}, \quad \operatorname{Pr}=\frac{\rho \mathrm{v} C_{p}}{K T}, \quad R=\frac{4 \mathrm{v} I^{\prime}}{\rho C_{p} U_{0}^{2}}, \quad \mathrm{Sc}=\frac{\mathrm{v}}{D}, \quad K c=\frac{K_{c}^{\prime} \mathrm{v}}{U_{0}^{2}}, \\
& \text { So }=\frac{D_{1}\left(T_{w}^{\prime}-T_{\infty}^{\prime}\right)}{v\left(C_{w}^{\prime}-C_{\infty}^{\prime}\right)}, \quad h=\frac{L_{l} U_{0}^{2}}{v}
\end{aligned}
$$

\section{Solution of the problem}

To solve the partial differential Eqs (2.9), (2.10) and (2.11), we reduce them into ordinary differential equations. To obtain the solution we follow the procedure given by Gersten and Gross. Therefore the expressions for velocity, temperature and concentration are assumed in the following form.

$$
\begin{aligned}
& U(y, t)=f_{0}(y)+\varepsilon f_{l}(y) e^{-n t}, \\
& T(y, t)=g_{0}(y)+\varepsilon g_{1}(y) e^{-n t}, \\
& C(y, t)=h_{0}(y)+\varepsilon h_{1}(y) e^{-n t} .
\end{aligned}
$$


Substituting the above expressions (3.1) in to Eqs (2.9), (2.10), (2.11) and equating the coefficient of $\varepsilon^{0}, \varepsilon^{l}$ (neglecting $\varepsilon^{2}$ terms etc.,), we obtain the following set of ordinary differential equations

$$
\begin{aligned}
& f_{0}^{\prime \prime}+f_{0}^{\prime}-M_{1} f_{0}=-\operatorname{Gr} g_{0}-\mathrm{Gm} h_{0}, \\
& f_{1}^{\prime \prime}+f_{1}^{\prime}+M_{2} f_{1}=-\operatorname{Gr} g_{1}-\mathrm{Gm} h_{1}-f_{0}^{\prime}, \\
& g_{0}^{\prime \prime}+\operatorname{Pr} g_{0}^{\prime}-R \operatorname{Pr} g_{0}=0, \\
& g_{1}^{\prime \prime}+\operatorname{Pr} g_{1}^{\prime}+\left(\frac{n \operatorname{Pr}}{4}-R \operatorname{Pr}\right) g_{1}=-\operatorname{Pr} g_{0}^{\prime}, \\
& h_{0}^{\prime \prime}+\operatorname{Sc} h_{0}^{\prime}-K_{c} \operatorname{Sc} h_{0}=-\operatorname{Sc} S_{0} g_{0}^{\prime \prime}, \\
& h_{1}^{\prime \prime}+\operatorname{Sc} h_{1}^{\prime}+\left(\frac{n \operatorname{Sc}}{4}-\operatorname{Sc} K_{c}\right) h_{1}=\operatorname{Sc} S_{0} g_{1}^{\prime \prime}-\operatorname{Sc} h_{0}^{\prime} .
\end{aligned}
$$

Here $\quad M_{2}=\frac{n}{4}-M_{1}$,

and the boundary conditions (12) reduce to

$$
\begin{aligned}
& u_{0}=h u_{0}^{\prime}, \quad u_{1}=h u_{1}^{\prime}, \quad T_{0}=1, \quad T_{1}=0, \quad C_{0}=1, \quad C_{1}=0 \quad \text { at } \quad y=0, \\
& u_{0} \rightarrow 0, \quad u_{1} \rightarrow 0, \quad T_{0} \rightarrow 0, \quad T_{1} \rightarrow 0, \quad C_{0} \rightarrow 0, \quad C_{1} \rightarrow 0 \quad \text { at } \quad y \rightarrow \infty .
\end{aligned}
$$

Equations (3.2) to (3.7) are second order linear differential equations with constant coefficients. The solutions of these paired equations under the corresponding boundary conditions (3.8) are

$$
\begin{aligned}
& g_{0}=e^{-m_{1} y}, \\
& g_{1}=A_{1}\left(e^{-m_{1} y}-e^{-m_{2} y}\right), \\
& h_{0}=e^{-m_{3} y}\left(1-A_{2}\right)+A_{2} e^{-m_{1} y}, \\
& h_{1}=\left(-A_{3}-A_{4}-A_{5}\right) e^{-m_{4} y}+A_{3} e^{-m_{1} y}+A_{4} e^{-m_{2} y}+A_{5} e^{-m_{3} y}, \\
& f_{0}=A_{6} e^{-m_{1} y}+A_{7} e^{-m_{3} y}+A_{8} e^{-m_{5} y}, \\
& f_{1}=A_{9} e^{-m_{1} y}+A_{10} e^{-m_{2} y}+A_{11} e^{-m_{3} y}+A_{12} e^{-m_{4} y}+A_{13} e^{-m_{5} y}+A_{14} e^{-m_{6} y} .
\end{aligned}
$$


Introducing Eqs (3.9) to (3.14) in Eqs (3.1), we get the expressions for velocity, temperature and concentration as

$$
\begin{aligned}
& U(y, t)=\left(A_{6} e^{-m_{1} y}+A_{7} e^{-m_{3} y}+A_{8} e^{-m_{5} y}\right)+\varepsilon\left(A_{9} e^{-m_{1} y}+A_{10} e^{-m_{2} y}+\right. \\
& \left.+A_{11} e^{-m_{3} y}+A_{12} e^{-m_{4} y}+A_{13} e^{-m_{5} y}+A_{14} e^{-m_{6} y}\right) e^{-n t}, \\
& T(y, t)=\left(e^{-m_{1} y}\right)+\varepsilon A_{1}\left(e^{-m_{1} y}-e^{-m_{2} y}\right) e^{-n t}, \\
& C(y, t)=\left(1-A_{2}\right) e^{-m_{3} y}+A_{2} e^{-m_{1} y}+ \\
& +\varepsilon\left(\left(-A_{3}-A_{4}-A_{5}\right) e^{-m_{4} y}+A_{3} e^{-m_{1} y}+A_{4} e^{-m_{2} y}+A_{5} e^{-m_{3} y}\right) e^{-n t} .
\end{aligned}
$$

\section{Skin friction}

The expression for the skin-friction $(\tau)$ at the plate is

$$
\tau=\left(\frac{d U}{d y}\right)_{y=0}=\left(\frac{d f_{0}}{d y}\right)_{y=0}+\varepsilon\left(\frac{d f_{1}}{d y}\right)_{y=0} e^{-n t}=A_{15}+\varepsilon A_{16} e^{-n t}
$$

\section{Rate of heat transfer}

The expression for the rate of heat transfer in terms of the Nusselt number $\left(\mathrm{N}_{\mathrm{u}}\right)$ is

$$
\mathrm{Nu}=\left(\frac{d T}{d y}\right)_{y=0}=\left(\frac{d g_{0}}{d y}\right)_{y=0}+\varepsilon\left(\frac{d g_{1}}{d y}\right)_{y=0} e^{-n t}=-m_{1}+\varepsilon A_{17} e^{-n t} .
$$

\section{Rate of mass transfer}

The expression for the rate of heat transfer in terms of the Sherwood number (Sh) is

$$
\mathrm{Sh}=\left(\frac{d C}{d y}\right)_{y=0}=\left(\frac{d h_{0}}{d y}\right)_{y=0}+\varepsilon\left(\frac{d h_{1}}{d y}\right)_{y=0} e^{-n t}=A_{18}+\varepsilon A_{19} e^{-n t} .
$$

\section{Results and discussion}

To assess the physical depth of the problem, the effects of various parameters such as the slip parameter $\mathrm{h}$, Grashof number Gr, magnetic parameter $M$, permeability of porous medium $K$, heat source parameter $H$, radiation parameter $R$, chemical reaction parameter $K r$, modified Grashof number Gm and Schimidt number Sc on velocity distribution, temperature distribution and concentration distribution are studied in Figs 1 to 10, while keeping the other parameters as constants. Figure 1 depicts the velocity profiles with the variations in $h$, it is observed that the significance of the velocity is high near the plate and thereafter it decreases and reaches to the stationery position at the other side of the plate. As expected the velocity increases with an increase in $h$. The effects of $\mathrm{Gr}$ on the velocity distribution are presented in Fig.2. In Fig.3 the velocity increases as $\mathrm{Gm}$ increases. From this figure it is noticed that the velocity increases with an 
increase in Gm. In Fig.4 the effects of $M$ on the velocity are shown. From this figure it is noticed that velocity decreases as $M$ increases. The applied magnetic field acts as Lorentz's force which drags the velocity. In Fig.5 the velocity increases as $K$ increases. In Figs 6 and 9 it is observed that the temperature decreases as $R$ and Pr increases. From Fig. 7 it is observed that the temperature decreases as Sc increases. Similarly, in the Fig. 8 the concentration decreases as $K c$ increase respectively, and in Fig.10 the concentration increases with an increase in So.

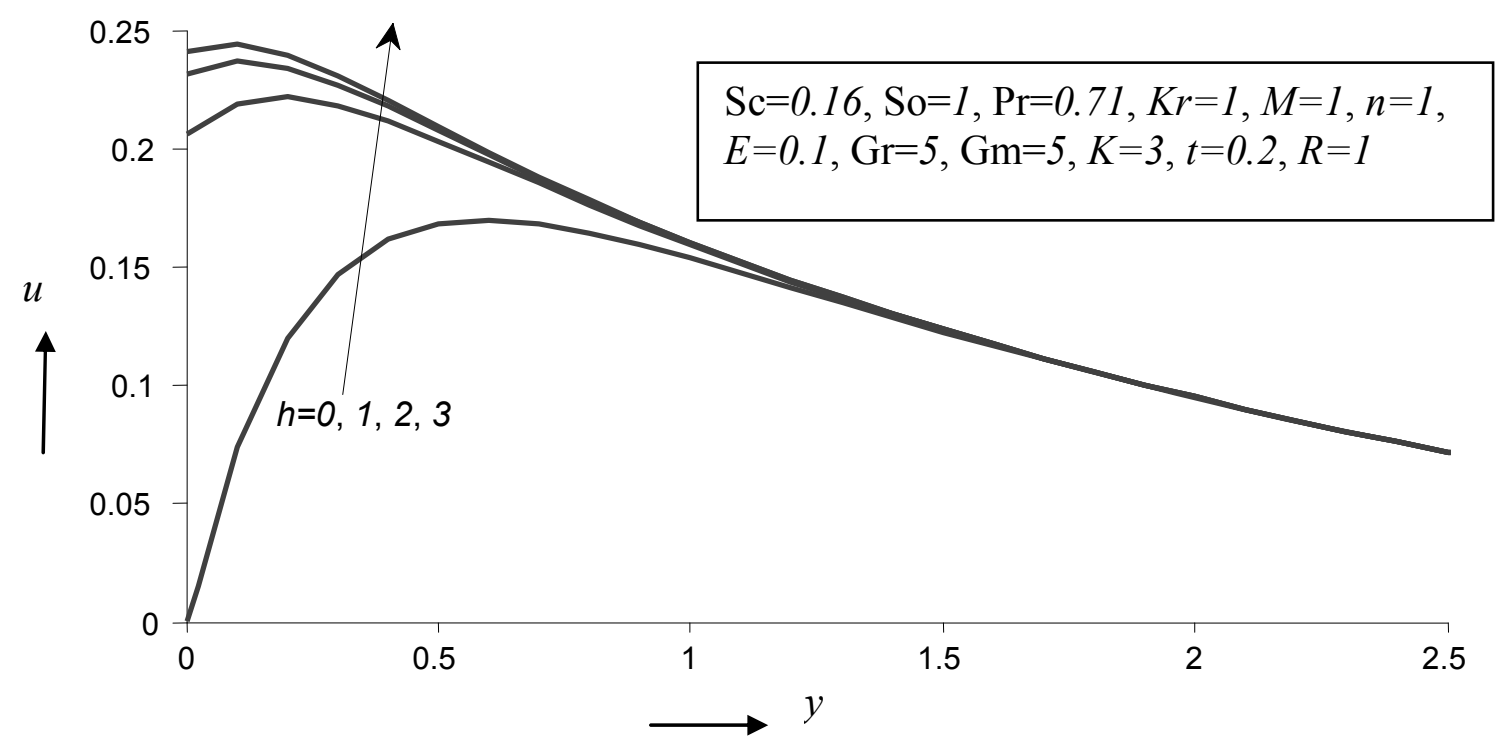

Fig.1. Effects of slip parameter $(h)$ on velocity.

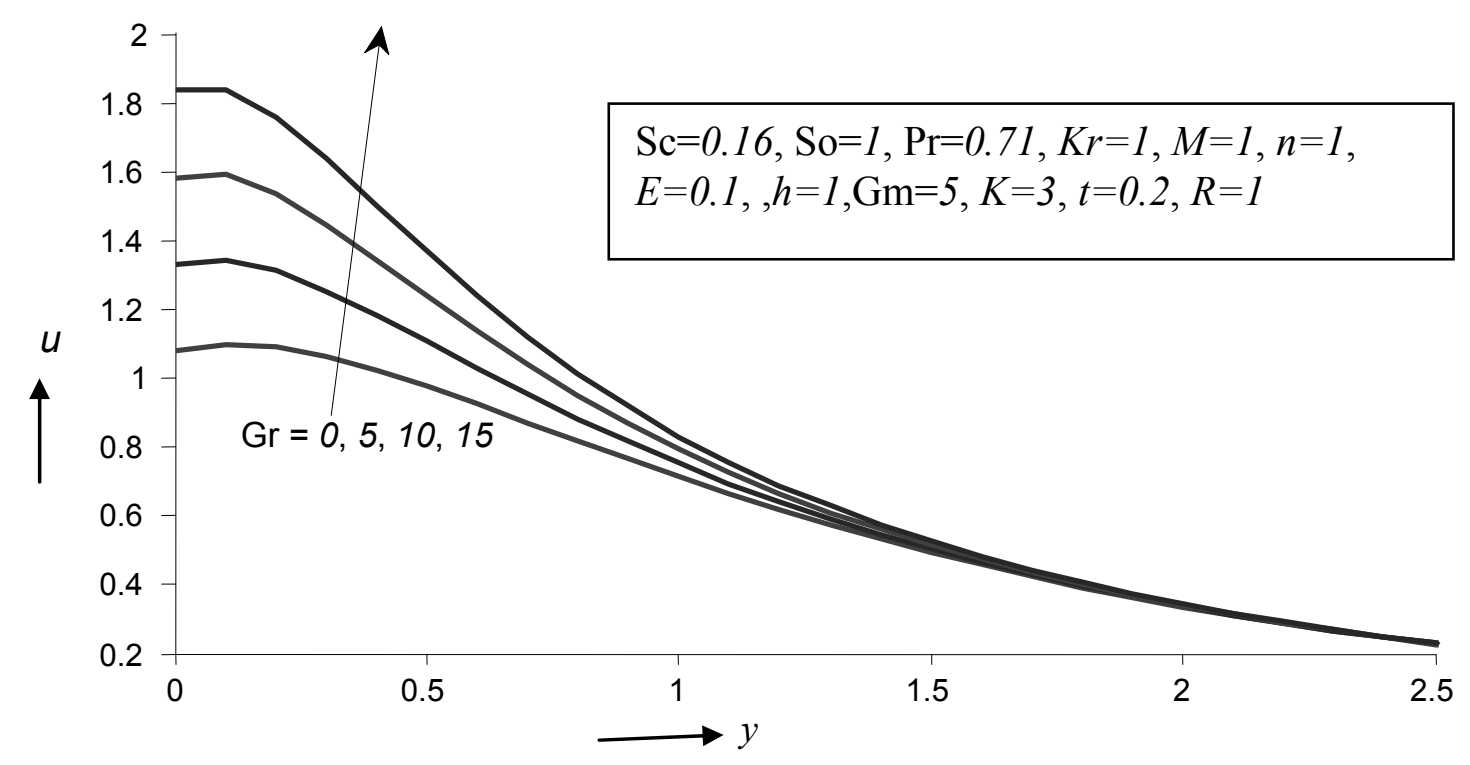

Fig.2. Effects of the Grashof number (Gr) on velocity. 


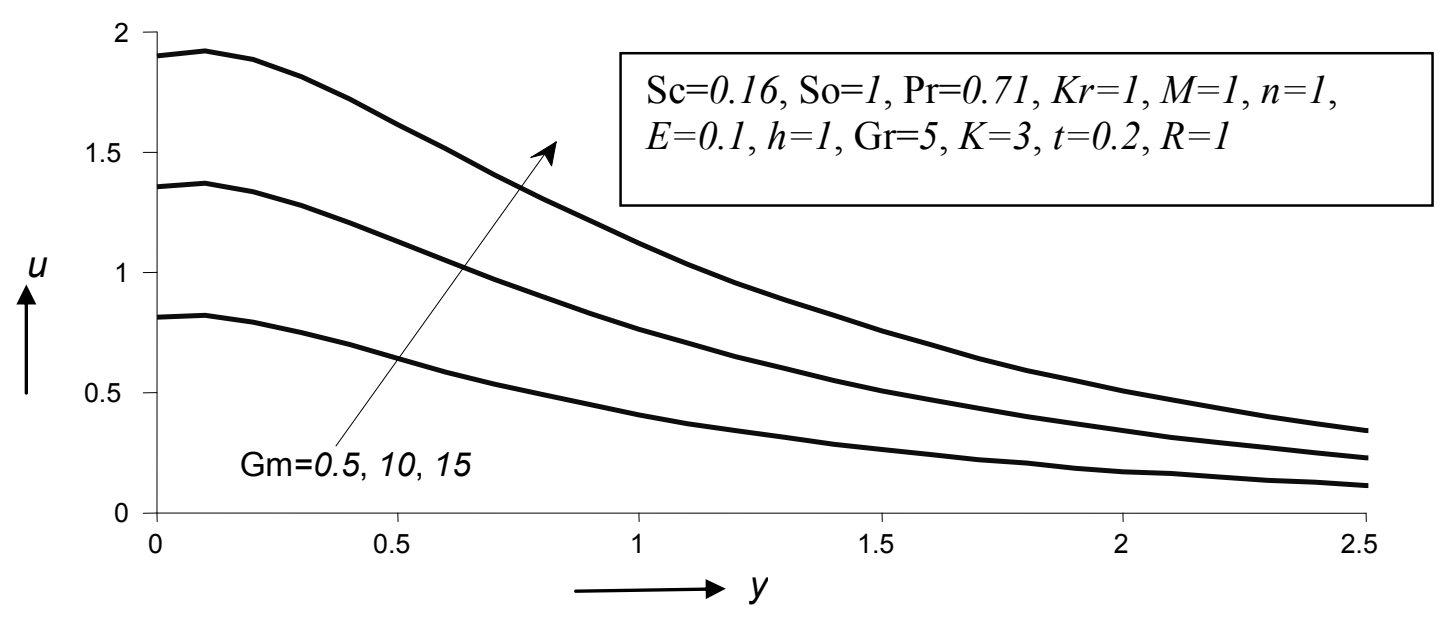

Fig.3. Effects of modified Grashof number (Gm) on velocity.

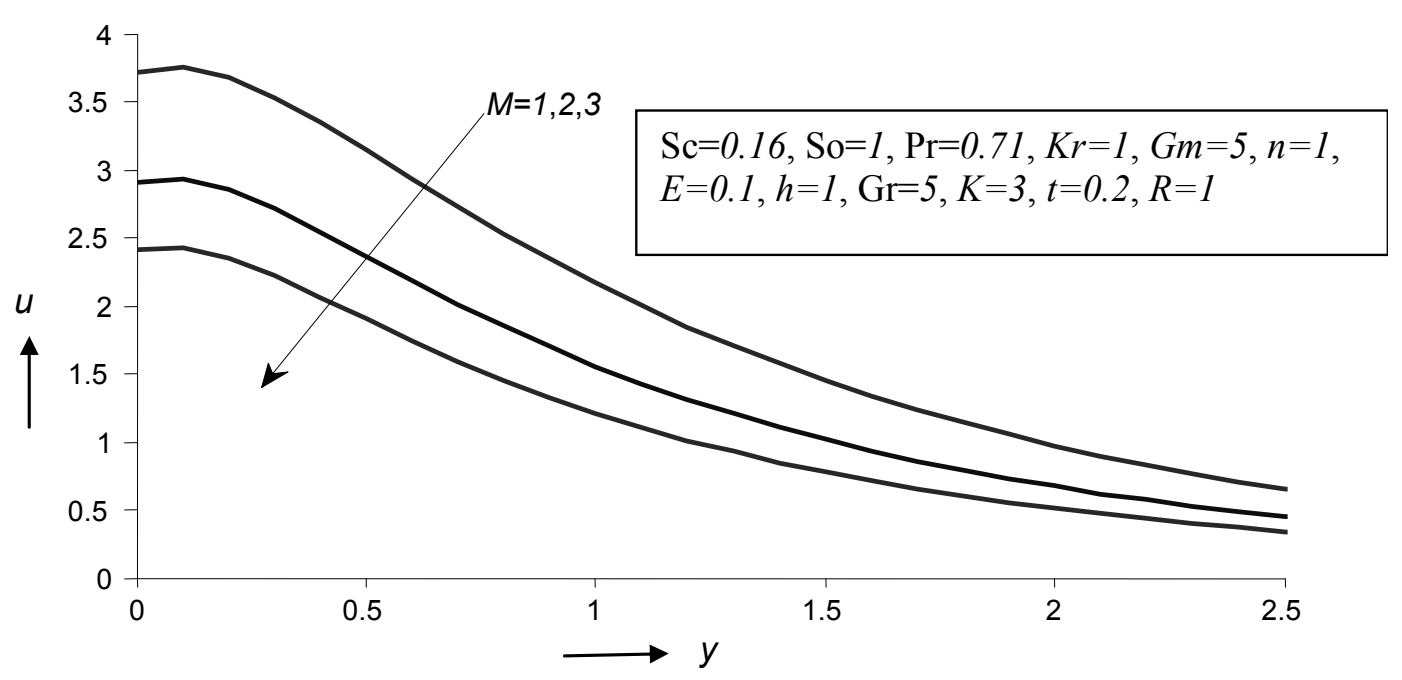

Fig.4. Effects of magnetic parameter $(M)$ on velocity.

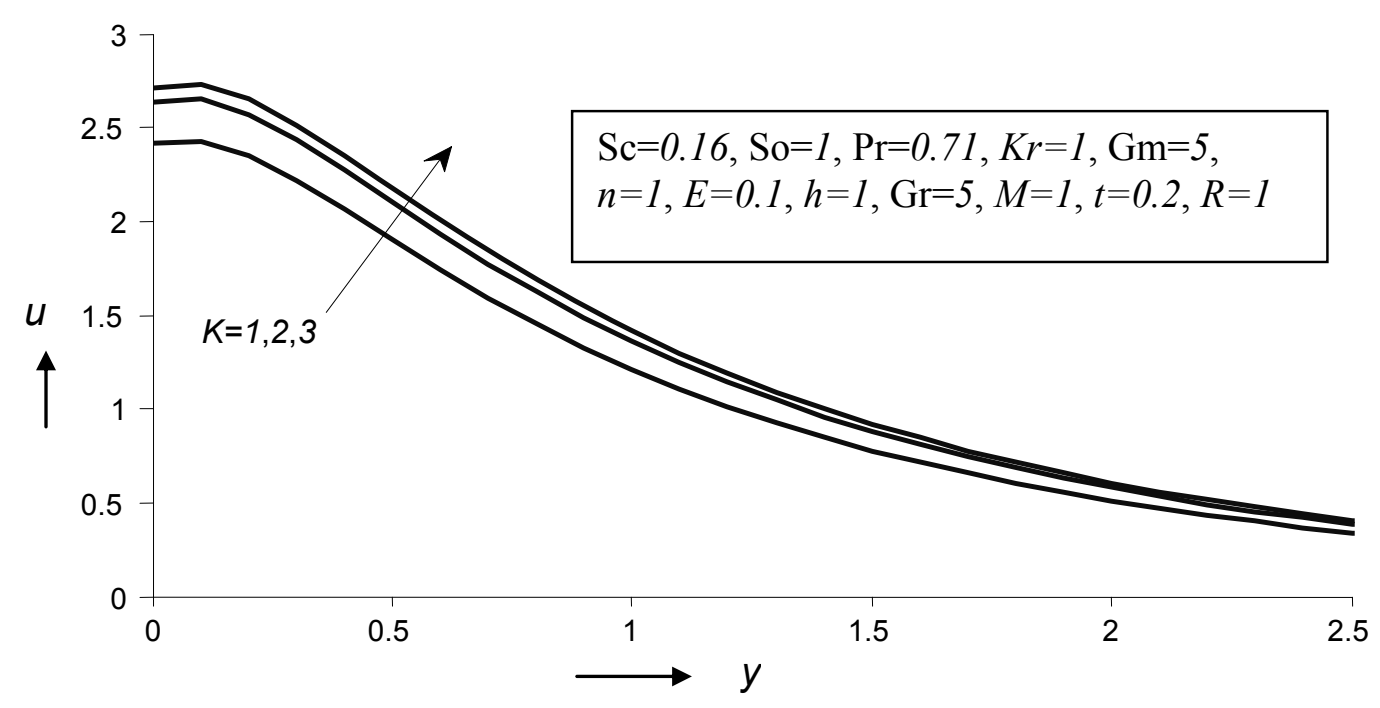

Fig.5. Effects of permeability parameter $(K)$ on velocity. 


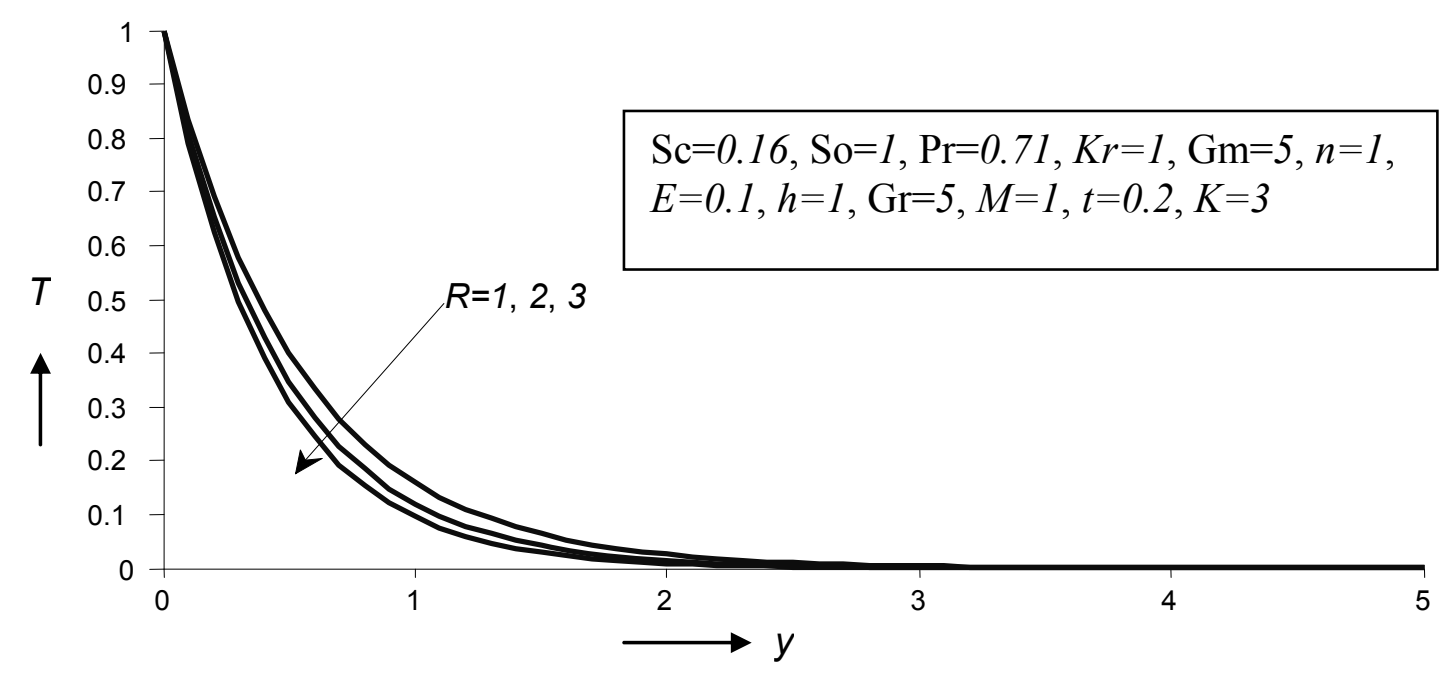

Fig.6. Effects of radiation parameter $(R)$ on temperature.

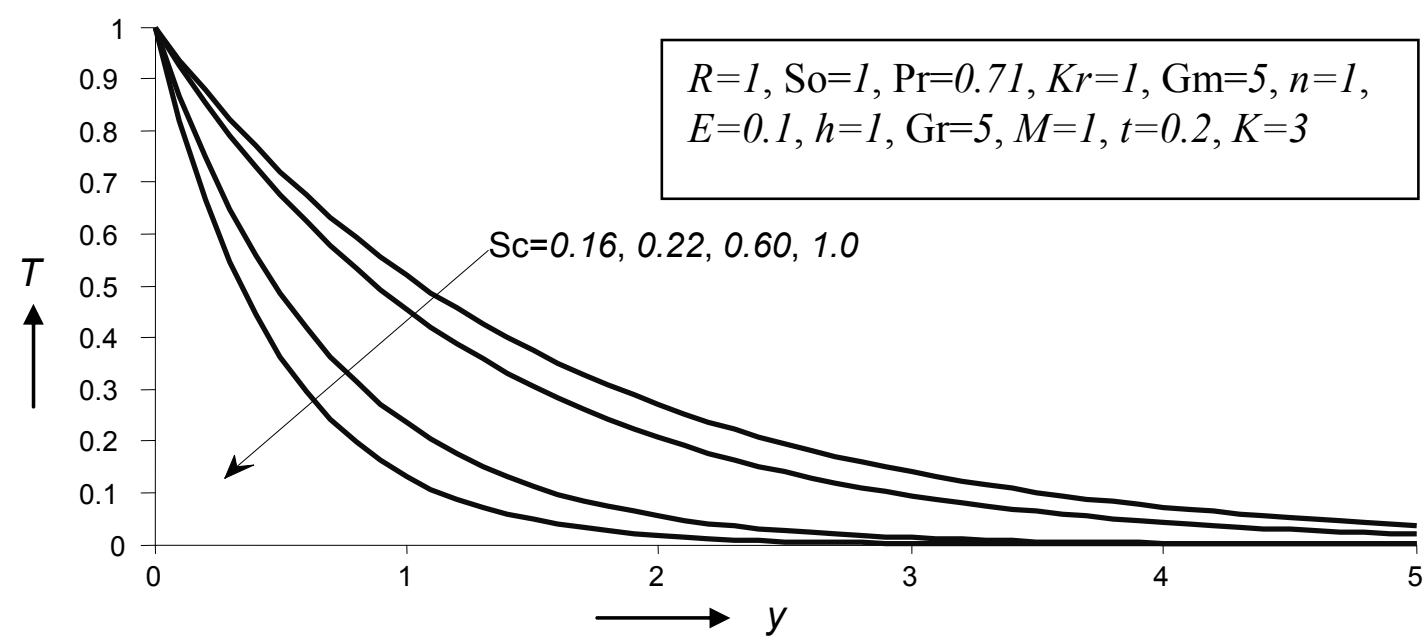

Fig.7. Effects of Schmidt number (Sc)on concentration.

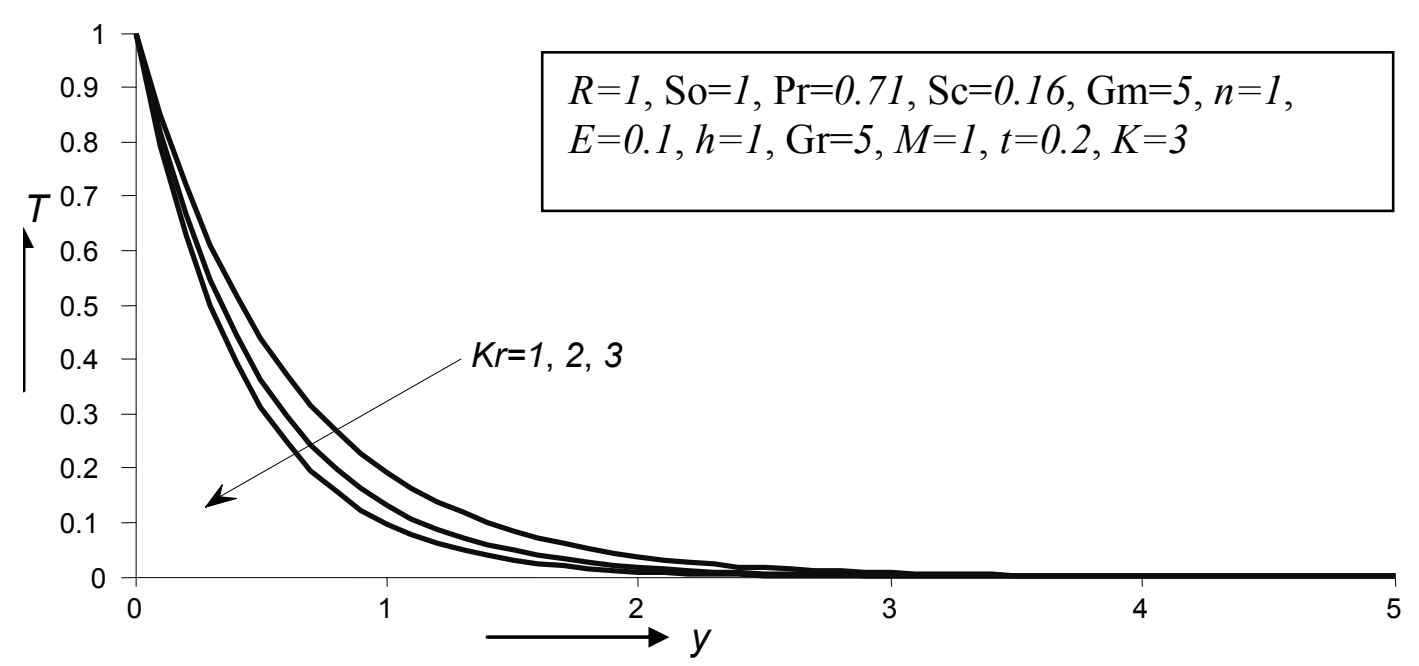

Fig.8. Effects of chemical reaction parameter $(\mathrm{Kr})$ on concentration. 


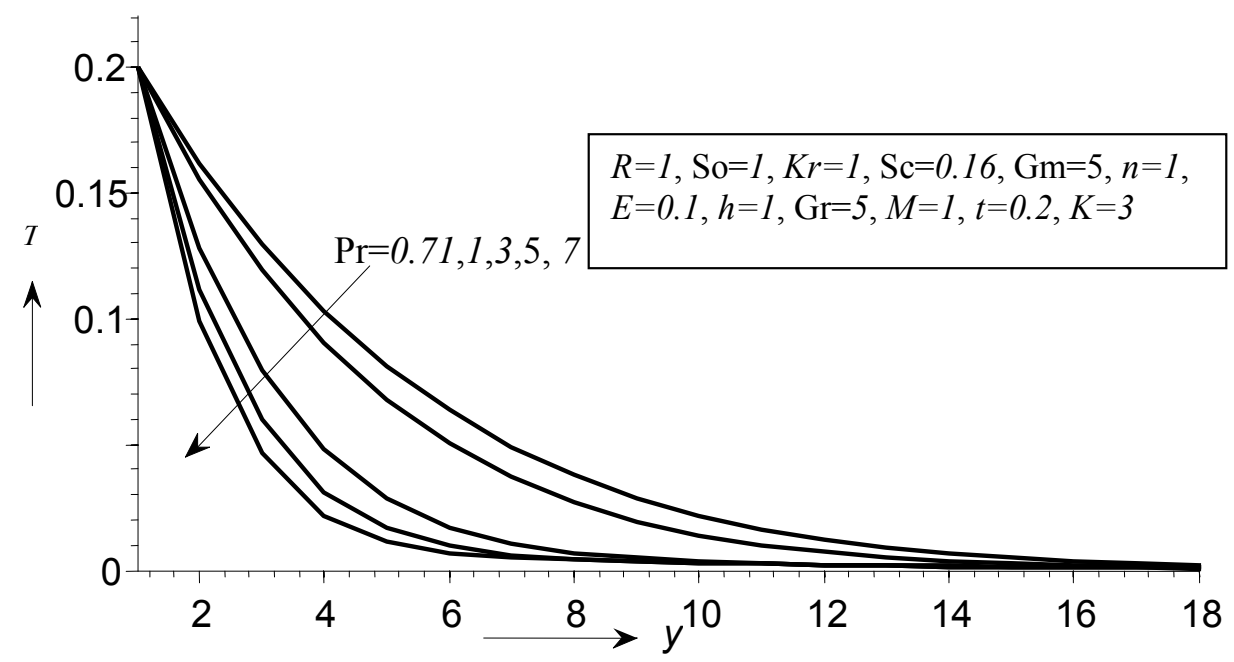

Fig.9. Effect of the Prandtl number (Pr) on temperature.

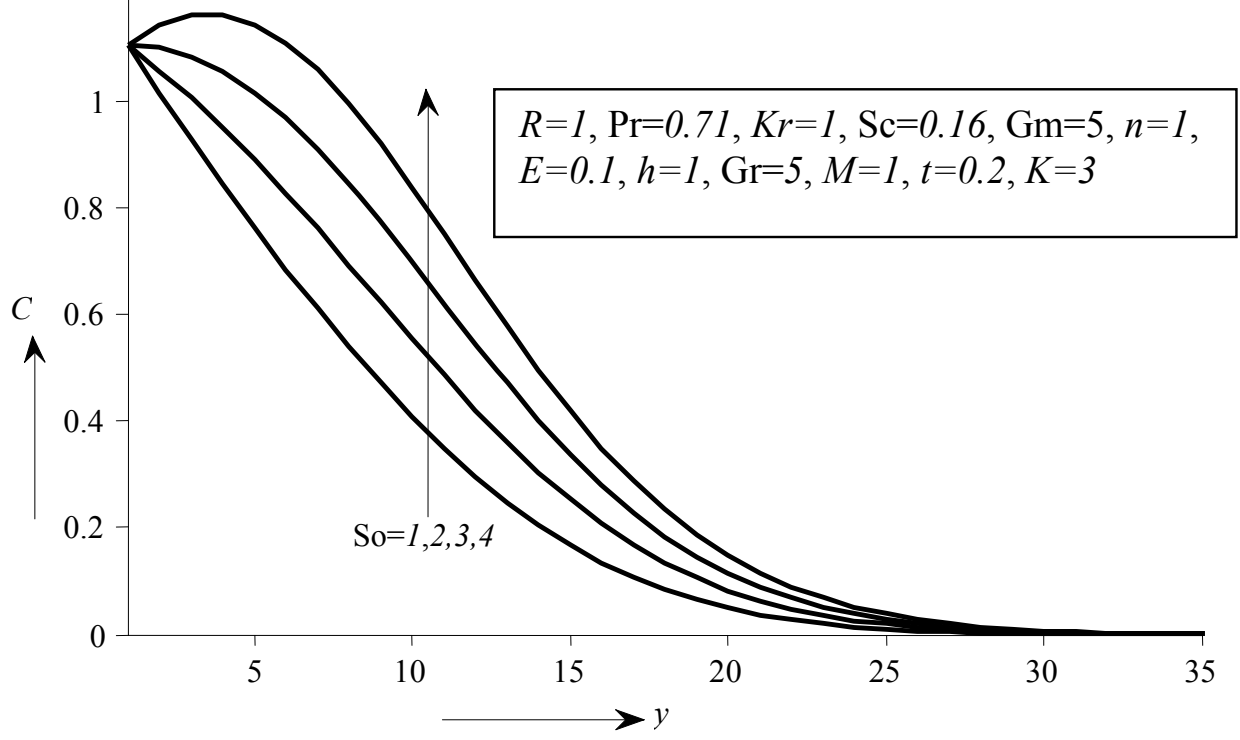

Fig.10. Effects of the Soret number (So) on concentration.

\section{Conclusions}

- $\quad$ The velocity increases with an increase in the slip parameter, Grashof number, modified Grashof number, permeability of porous medium and it shows a reverse effect with an increase in the magnetic parameter.

- $\quad$ The temperature decreases with an increase in the radiation parameter and the Prandtl number.

- The concentration decreases with an increase in the Schmidt number and chemical reaction parameter and shows a reverse effect with an increase in the Soret effect. 


\section{Appendix}

$m_{1}=\frac{\mathrm{Sc}+\sqrt{\mathrm{Sc}^{2}+4 K_{c} \mathrm{Sc}}}{2}, \quad m_{2}=\frac{\mathrm{Sc}+\sqrt{\mathrm{Sc}^{2}+\mathrm{Sc}\left(4 K_{c}-n\right)}}{2}, \quad m_{3}=\frac{(1+H) \operatorname{Pr}+\sqrt{(1+H)^{2} \operatorname{Pr}^{2}+4 R \operatorname{Pr}}}{2}$,

$m_{4}=\frac{(1+H) \operatorname{Pr}+\sqrt{(1+H)^{2} \operatorname{Pr}^{2}+(4 R-n) \operatorname{Pr}}}{2}, \quad m_{5}=\frac{1+\sqrt{1+4 M_{1}}}{2}, \quad m_{6}=\frac{1+\sqrt{1+4 M_{2}}}{2}$,

$A_{1}=\frac{\operatorname{Sc} m_{1}}{m_{1}^{2}-\operatorname{Sc} m_{1}-\left(K_{c}-\frac{n}{4}\right) \mathrm{Sc}}, \quad A_{2}=\frac{\operatorname{Pr} m_{3}}{m_{3}^{2}-(1+H) \operatorname{Pr} m_{3}-\left(R-\frac{n}{4}\right) \operatorname{Pr}}, \quad A_{3}=-\frac{\mathrm{Gr}}{m_{3}^{2}-m_{3}-M_{1}}$,

$A_{4}=-\frac{\mathrm{Gm}}{m_{1}^{2}-m_{1}-M_{1}}, \quad A_{5}=-\left(\frac{A_{3}\left(1+h m_{3}\right)+A_{4}\left(1+h m_{1}\right)}{1+h m_{5}}\right), \quad A_{6}=\frac{\left(A_{5} m_{5}-\frac{A_{5}}{K_{0}}\right.}{m_{5}{ }^{2}-m_{5}-M_{2}}$,

$A_{7}=\frac{\left(A_{3} m_{3}-A_{2} \mathrm{Gr}-\frac{A_{3}}{K_{0}}\right)}{m_{3}{ }^{2}-m_{3}-M_{2}}, \quad A_{8}=\frac{\left(A_{4} m_{1}-A_{1} \mathrm{Gm}-\frac{A_{4}}{K_{0}}\right)}{m_{1}{ }^{2}-m_{1}-M_{2}}, A_{9}=\frac{A_{2} \mathrm{Gr}}{m_{4}{ }^{2}-m_{4}-M_{2}}, A_{10}=\frac{A_{1} \mathrm{Gm}}{m_{2}{ }^{2}-m_{2}-M_{2}}$,

$A_{11}=-\left(\frac{A_{6}\left(1+h m_{5}\right)+A_{7}\left(1+h m_{3}\right)+A_{8}\left(1+h m_{1}\right)+A_{9}\left(1+h m_{4}\right)+A_{10}\left(1+h m_{2}\right)}{1+h m_{6}}\right)$,

$A_{12}=-A_{5} m_{5}-A_{3} m_{3}-A_{4} m_{1}, \quad A_{13}=-A_{11} m_{6}-A_{6} m_{5}-A_{7} m_{3}-A_{8} m_{1}-A_{9} m_{4}-A_{10} m_{2}$,

$A_{14}=A_{2} m_{4}-A_{2} m_{3}, \quad A_{15}=A_{1} m_{2}-A_{1} m_{1}$.

\section{Nomenclature}
$a$ - constant
$C$ - concentration
$C_{p}$ - specific heat at constant pressure
$D$ - molecular diffusivity
$D_{l}$ - thermal diffusivity
Df - Dufour number
Gm - modified Grashof number
$\mathrm{Gr}$ - Grashof number
$g$ - acceleration due to gravity
$K$ - porosity parameter
$K_{P}$ - permeability of the medium
$K r$ - chemical reaction parameter
$k$ - thermal diffusivity
$M$ - magnetic parameter
$\mathrm{Nu}$ - Nusselt number 
Pr - Prandtl number

$Q$ - heat absorption parameter

$R$ - radiation parameter

Sc - Schmidt number

Sh - Sherwood number

So - Soret number

$t$ - time

$u$ - velocity of the fluid

$y$ - coordinate axis normal to the plate

$\beta-$ volumetric coefficient of thermal expansion

$\beta^{*}-$ volumetric coefficient of concentration expansion

$\theta-$ temperature

$\mu-$ coefficient of viscosity

$v$ - kinematic viscosity

$\rho-$ density of the fluid

$\sigma$ - electrical conductivity

$\tau$ - skin friction

$\chi$ - radiation absorption parameter

\section{References}

[1] Chamkha A.J. (2000): Hydro-magnetic combined heat and mass transfer by natural convection from a permeable surface embedded in a fluid saturated porous medium. - Int. J. Num. Methods for Heat and Fluid Flow, vol.10, No.5, pp.455-476.

[2] Chamkha A.J. and Khaled A.R. (2000): Hydro-magnetic combined heat and mass transfer by natural convection from a permeable surface embedded in fluid saturated porous medium. - International Journal of Numerical Methods for Heat and Fluid Flow, vol.10, No.5, pp.455-476.

[3] Chamkha A.J. (2003): MHD flow of a uniformly stretched vertical permeable surface in the presence of heat generation/absorption and a chemical reaction. - Int. Commun. Heat Mass Transfer, vol.30, No.3, pp.413-422.

[4] Kandasamy R., Periasamy K. and Prabhu K.K.S. (2005): Chemical reaction, heat and mass transfer on MHD flow over a vertical stretching surface with heat source and thermal stratification effects. - Int. J. Heat Mass Transfer, vol.48, pp.4557-4561.

[5] Postelnicu A. (2007): Influence of chemical reaction on heat and mass transfer by natural convection from vertical surfaces in porous media considering Soret and Dufour effects. - Heat and Mass Transfer, vol.43.

[6] Hossain A.M., Alim M.A. and Rees D.A.S. (1999): Effect of radiation on free convection from a porous a vertical plate. - International Journal of Heat and Mass Transfer, vol.42, pp.181-191.

[7] Ibrahim F.S., Elaiw A.M. and Bakr A.A. (2008): Effect of the chemical reaction and radiation absorption on the unsteady MHD free convection flow past a semi infinite vertical permeable moving plate with heat source and suction. - Comm. Nonlinear Sci. Numer. Simul., vol.13, No.6, pp.1056-1066.

[8] Sharma P.K. and Chaudary R.C. (2003): Effect of variable suction on transient free convection viscous incompressible flow past a vertical plate with periodic temperature variations in slip-flow regime. - Emirates J. Engineering Research, vol.8, pp.33-38.

[9] Sharma P.K. (2005): Fluctuating thermal and mass diffusion on unsteady free convective flow past a vertical plate in slip-flow regime. - Latin American Applied Research, vol.35, pp.313-319.

[10] Pal D. and Talukdar B. (2009): Perturbation analysis of unsteady magneto hydrodynamic convective heat and mass transfer in a boundary layer slip flow past a vertical permeable plate with thermal radiation and chemical reaction. - Communications in Nonlinear Science and Numerical Simulation, In Press, Corrected Proof, Available online 17 July 2009. 
[11] Kumar K., Varshney M. and Varshney C.L. (1987): Elastic-viscous stratified fluctuating Hartmann flow through a porous medium past an infinite rigid plane in slip regime. - JMACT, vol.20, pp.65-71.

[12] Muthukumaraswamy R. and Kumar G.S. (2004): Heat and mass transfer effects on moving vertical plate in the presence of thermal radiation. - Theoret. Appl. Mach., vol.31, No.1, pp.35-46.

[13] Gupta M. and Sharma S. (1991): MHD flow of viscous fluid through a porous medium bounded by an oscillating porous plate in slip flow regime. - Acta Ciencia Indica, vol.17M, pp.389-394.

[14] Singh Atul Kumar, Singh N.P. and Singh R.V. (2000): MHD Flow of a dusty visco-elastic liquid (Rivlin-Ericksen) through a porous medium bounded by an oscillating porous plate in slip flow regime. - The Math. Edu., vol.34, pp.53-55.

[15] Singh N.P., Singh R.V. and Singh Atul Kumar (1998): Flow of a dusty visco- elastic fluid through a porous medium near an oscillating porous plate in slip flow regime. - JMACT, vol.31, pp.99-108.

[16] Singh N.P., Singh R.V. and Singh Atul Kumar (1999): Flow of a visco-elastic fluid through a porous medium near an oscillating plate in slip flow regime in the presence of electromagnetic field. - Ind. J. Theo. Phys., vol.47, pp.203-209.

[17] Jaiswal B.S. and Soundalgekar V.M. (2000): Transient convective flow past an infinite vertical oscillating plate with temperature gradient dependent heat source. - Bul. All India Math. Soc., vol.15, pp.25-29.

[18] Taneja R. and Jain N.C. (2002): Effects of magnetic field on free convection and mass transfer flow through porous medium and variable permeability in slip flow regime. - Jnanabh, vol.31/32, pp.69-81.

[19] Yamamoto K. and Yoshidha Z. (1974): Flow through a porous wall with convective acceleration. - J. Phys. Soc., Japan, vol.37, pp.774-786.

[20] Yamamoto K. and Iwamura N. (1976): Flow with convective acceleration through a porous medium. - J. Engg. Math., vol.10, pp.41-54.

[21] Raju M.C., Varma S.V.K. and Reddy N.A. (2011): MHD thermal diffusion natural convection flow between heated inclined plates in porous medium. - Journal on Future Engineering and Technology, vol.6, No.2, pp.4548.

[22] Raju M.C., Varma S.V.K. and Reddy N.A. (2012): Radiation and mass transfer effects on a free convection flow through a porous medium bounded by a vertical surface. - Journal of Future Engineering and Technology, vol.7, No.2, pp.7-12.

[23] Raju M.C., Varma S.V.K., Reddy P.V. and Sumon Saha (2008): Soret effects due to natural convection between heated inclined plates with magnetic field. - Journal of Mechanical Engineering, vol.ME39, pp.43-48.

[24] Kim J. and Youn (2000): Unsteady MHD convective heat transfer flow past a semi-infinite vertical porous moving plate with variable suction. - International Journal of Engineering Sciences, vol.38, pp.833-845.

[25] Daniel P.G., Blythe B.A. and Simpkins P.G. (1985): Thermally driven shallow cavity flows in porous medium for the intermediate regime. - Proc. Roy. Soc., London, vol.406, pp.263-285.

[26] Ede A.J. (1967): Advances in Heat Transfer Academic Press. - New York.

Received: January 5, 2018

Revised: November 14, 2018 